\title{
(6) OPEN ACCESS \\ Can lumbar spine bone mineral density predict readmission in denosumab-treated patients with chronic kidney disease?
}

\author{
Ben-Chung Cheng, ${ }^{1,3}$ Ying-Chou Chen ${ }^{2}$
}

${ }^{1}$ Department of Nephrology, Kaohsiung Chang Gung Memorial Hospital, Chang Gung University College of Medicine, Kaohsiung, Taiwan

${ }^{2}$ Department of Rheumatology, Kaohsiung Chang Gung Memorial Hospital, Chang Gung University College of Medicine, Kaohsiung, Taiwan

${ }^{3}$ Department of Biological Science, National Sun YatSen University, Kaohsiung, Taiwan

\section{Correspondence to} Dr Ying-Chou Chen, Department of Rheumatology, Kaohsiung Chang Gung Memorial Hospital, Chang Gung University College of Medicine, 123 Ta-Pei Road, Niao-Sung Dist., Kaohsiung 833, Taiwan; r820713@ ms13.hinet.net

Accepted 3 August 2016 Published Online First 31 August 2016

Copyright (C) 2016 American Federation for Medical Research

\section{ABSTRACT}

This study investigated whether bone mineral density (BMD) affects readmission risk in patients with chronic kidney diseases (CKD) who received denosumab therapy. The study design was a retrospective case review of patients with CKD. Baseline age, sex, and body mass index were recorded for all patients included in the study. All comorbidities were recorded. All subjects underwent dual energy $X$-ray absorptiometry assay of the lumbar spine and right hip for BMD. The primary outcome was readmission. Predictive variables were categorized and compared between readmitted and non-readmitted patients. Logistic regression was used for multivariable analysis. A total of 121 patients with CKD who received denosumab therapy were enrolled. Of these, 29 were readmitted within 2 years, and 92 had no readmission. The lumbar BMD differed between the readmission $(-2.94$ $\pm 0.68)$ and non-readmission $(-2.09 \pm 1.48)$ groups. The readmission group had a lower $\mathrm{T}$ score than the non-readmission group. When adjusted for potential confounding factors, a decreased lumbar BMD had a higher readmission risk. When the cut-off points determined by receiver operating characteristic curve analysis were applied, the most precise point was set at a $T$ score of -3 . Osteoporosis in patients with CKD is associated with a high risk of readmission; the best predictor after denosumab therapy was the lumbar spine T score. A lower T score (especially if $<-3$ ) was associated with a higher probability of fracture readmission. It is essential to optimize primary and secondary prevention in these patients to improve their quality of life.

\section{BACKGROUND}

Bone disease in chronic kidney disease (CKD) is common and may result in fractures associated with substantial morbidity and mortality. Patients with advanced CKD stages had increased risk of fracture than general population. ${ }^{1}$ In patients with CKD, abnormal bone turnover leads to accelerated bone loss and a higher prevalence of osteopenia, osteoporosis, etc. ${ }^{2}$

The qualitative abnormalities of low bone mass in CKD usually reflected by the high incidence of fragility fractures. ${ }^{1}{ }^{3}$ Hence, it is important to regularly evaluate bone mineral density (BMD) in these patients. Dual energy X-ray absorptiometry (DXA) is suggested and

\section{Significance of this study}

What is already known about this subject?

- The rapid increased incidence and prevalence of chronic kidney diseases (CKD) have been recognized as a global public health problem that consumes a large proportion of healthcare budgets.

- Bone disease in CKD is a common complication among a broad spectrum of disorders of mineral metabolism, and may result in fractures associated with substantial morbidity and mortality.

- Reports on the correlation of bone mineral density (BMD) values with fracture risk in the CKD population are inconsistent.

- Denosumab, a receptor activator of the nuclear factor $\kappa-\mathrm{B}$ ligand (RANKL) inhibitor, is an effective treatment for osteoporosis in postmenopausal women and men. Unlike the bisphosphonates, it is not excreted by the kidney.

\section{What are the new findings?}

- Osteoporosis in patients with CKD is associated with a high risk of readmission.

- The best predictor after denosumab therapy was the lumbar spine (LS) T score.

- A lower T score (especially $<-3$ ) was associated with a higher probability of fracture readmission.

How might these results change the focus of research or clinical practice?

- In patients with CKD, the risk of osteoporosis is higher, and the use of denosumab therapy will decrease fracture risk and mortality. Hence, LS BMD is the best predictor of fracture readmission in CKD. Aggressive medical attention is needed to decrease a future trend toward fracture readmissions.

reliable for evaluating osteoporosis in CKD patients based on BMD measurements applied to the lumbar spine or the femoral neck. ${ }^{2}$ Evidence has shown that bone loss in the FN increases the risk of FN fracture. Hence, FN BMD is considered the gold standard for patients with CKD. However, forearm BMD is 
also another quick and sensitive way to detect osteoporosis in patients with peritoneal dialysis. ${ }^{4}$ Yamaguchi et $a l^{5}$ found that distal radius is the preferred site for bone densitometry in CKD patients. However, other studies consider that the value of bone density measurement in the evaluation of CKD mineral and bone disorders is not well established. ${ }^{6}$

It is reported that the rate of kidney function decline is associated with a higher risk of hospitalization, readmission, and prolonged length of hospital stay. ${ }^{7}$

Denosumab, a receptor activator of the nuclear factor $\kappa-\mathrm{B}$ ligand (RANKL) inhibitor, is an effective treatment for osteoporosis in postmenopausal women and men. ${ }^{8-12}$ Unlike the bisphosphonates, it is not excreted by the kidney and is widely used in patients with CKD in Taiwan.

Little is known about whether BMD could predict readmission in denosumab-treated patients. Thus, this study investigated whether BMD affects readmission risk in patients with CKD who received denosumab therapy.

\section{MATERIALS AND METHODS}

The study design was a retrospective case review of patients with CKD treated with denosumab at Kaohsiung Chang Gang Memorial Hospital between January 2011 and December 2014. An Institutional Review Board at Kaohsiung Chang Gang Memorial Hospital approved the study, which was conducted in accordance with the Declaration of Helsinki and the International Conference on Harmonization of Good Clinical Practice Guidelines. In accord with Taiwanese law, no additional informed consent was required. Patient information was anonymized and de-identified before data analysis.

Baseline age, gender, and body mass index (BMI) were recorded for all patients included in the study. All comorbidities, such as diabetes, hypertension, and liver disease, as well as intact parathyroid hormone (iPTH) level were recorded.

All subjects underwent DXA assay of the lumbar spine (LS) and right hip for BMD by the same technologist, who was certified by the International Society of Clinical
Densitometry. The assay was performed using a fan beam bone densitometer (QDR-4500A; Hologic, Waltham, Massachusetts, USA). ${ }^{13}$ Instrument quality control was performed at least 5 days per week, and the coefficient of variation for the total BMD was $1.0 \%$. Based on the WHO definitions, normal BMD is within $1 \mathrm{SD}(+1$ or -1$)$ of the young adult mean, osteopenia is defined as BMD between 1 and 2.5 SD below the young adult mean $(-1$ to -2.5 $\mathrm{SD}$ ), and osteoporosis is BMD $2.5 \mathrm{SD}$ or more below the young adult mean $\left(-2.5 \mathrm{SD}\right.$ or lower). ${ }^{14}$ The primary outcome was defined as all-cause readmission within 2 years of following denosumab therapy. The secondary outcome was the reason for and the factors associated with readmission.

\section{Statistical analysis}

Statistical analysis was performed using SPSS software, V.21.0 (SPSS, Chicago, Illinois, USA). Patient characteristics were reported as simple descriptive statistics (mean $\pm \mathrm{SD}$ ). Comparisons between independent means were analyzed using the Student's t-test. Relationships between categorical variables were evaluated with the $\chi^{2}$ test. Logistical regression analysis was used to adjust for potential confounding factors. A $p$ value $<0.05$ was considered to be statistically significant.

\section{RESULTS}

A total of 121 patients with CKD who had received denosumab therapy were enrolled. Of these, 29 were readmitted within 2 years ( 9 hip fracture, 12 spine refracture, 6 knee surgery), and 92 had no readmission. There was no difference 2 herniation of intervertebral disc in age, BMI, gender, number of vertebral fractures, iPTH level, or underlying medical illness between the readmission and non-readmission groups. The CKD stages were also the same for the readmission and non-readmission groups $(\mathrm{p}=0.196)$. The BMD ( $\mathrm{T}$ score) at the FN among men was the same in the readmission and control groups. The lumbar BMD (T score) differed between the readmission $(-2.94 \pm 0.68)$ and nonreadmission $(-2.09 \pm 1.48)$ groups $(\mathrm{p}=0.005)$; the

Table 1 Characteristics of study patients

\begin{tabular}{llll}
\hline Variables & Readmission $(\mathbf{n = 2 9 )}$ & Non-readmission $(\mathbf{n = 9 2 )}$ & $\mathbf{p}$ Value \\
\hline Age (years) & $72.82 \pm 8.624$ & $74.77 \pm 6.77$ & 0.316 \\
Body mass index $\left(\mathrm{kg} / \mathrm{m}^{2}\right)$ & $23.80 \pm 3.45$ & $23.83 \pm 4.56$ & 0.977 \\
Spine fracture (number) & $1.72 \pm 1.77$ & $1.09 \pm 1.44$ & 0.052 \\
iPTH (pg/mL) & $188.27 \pm 305.04$ & $283.61 \pm 442.45$ & 0.41 \\
BMD (lumbar) & $-2.94 \pm 0.68$ & $-2.09 \pm 1.48$ & 0.005 \\
BMD (total hip) & $-1.91 \pm 0.96$ & $-1.75 \pm 1.15$ & 0.509 \\
BMD (femoral neck) & $-2.38 \pm 0.68$ & $-2.34 \pm 0.95$ & 0.84 \\
Gender (female \%) & $22(75.9 \%)$ & $78(84.8 \%)$ & 0.624 \\
Neurological disease & $5(17.2)$ & $12(13.0)$ & 0.384 \\
Diabetes mellitus (\%) & $10(34.5)$ & $24(26.1)$ & 0.478 \\
Hypertension & $21(72.4)$ & $55(59.8)$ & 0.273 \\
Hyperlipidemia & $2(6.9)$ & $4(4.3)$ & 0.629 \\
Heart disease & $8(27.6)$ & $18(19.6)$ & 0.437 \\
Pulmonary disease & $3(10.3)$ & $10(10.9)$ & 0.621 \\
Liver disease & $6(20.7)$ & $20(21.7)$ & 0.565 \\
\hline BMD, bone & m & & \\
\hline
\end{tabular}

$\mathrm{BMD}$, bone mineral density; iPTH, intact parathyroid hormone. 
readmission group had a lower BMD (table 1). There were 14 vertebral fractures, 12 hip fractures, 2 forearm fractures, and 1 knee fracture.

When adjusted for potential confounding factors such as diabetes, hypertension, pulmonary, and liver disease, a decreased lumbar BMD still had a higher readmission risk $(\mathrm{p}=0.007 ; \mathrm{OR}=1.96,95 \%$ CI 1.206 to 3.189$)$ (table 2), while age, gender, BMI, spine fracture, total hip BMD, and FN BMD did not increase the risk of readmission. When the cut-off points determined by receiver operating characteristic curve analysis were applied, the most precise cut-off point was set at a T score of -3 (sensitivity 0.630 ; specificity 0.788 ); patients with scores lower than -3 had a higher readmission risk.

\section{DISCUSSION}

The rapid increased incidence and prevalence of CKD have been recognized as a global public health problem that consumes a large proportion of healthcare budgets. ${ }^{15}$ In Taiwan, the national prevalence of CKD is high, and has the highest prevalence and incidence of end-stage renal disease in the world. ${ }^{16}$

Osteoporosis constitutes a significant worldwide burden on public health. In particular, patients with CKD have accelerated bone loss due to abnormal turnover that leads to a high prevalence of bone health problems. ${ }^{2}$ Treatment of patients at high risk of further fractures has proven to be effective in significantly reducing subsequent fractures. ${ }^{17}$ Among the antiosteoporotic drugs, denosumab blocks the formation, function, and survival of osteoclasts. ${ }^{10}$ The magnitude of risk reduction of vertebral fractures with denosumab was similar to that reported for intravenously administered zoledronic acid, and appears to be greater than that reported for oral osteoporosis agents. ${ }^{18}$ Denosumab increased BMD at the LS by about $9 \%$ and in the entire hip by about $6 \%$. Denosumab is not excreted in the kidney, and is widely used in patients with CKD in Taiwan.

Despite the high financial and social costs of readmission, there is a shortage of information about risk factors for fracture readmission after denosumab therapy in patients with CKD. There are multiple factors determining bone mass loss in patients with CKD, whose bone quality and quantity deteriorate rapidly. Although Gabay et $a l^{19}$ indicated that cortical bone is more sensitive than trabecular bone in CKD-related loss, baseline trabecular bone loss was more related to readmission in denosumab-treated patients in the present study. Decreased spine mass leads to more spinal fractures, kyphosis, and hip or other fractures; a greater tendency toward falls increases the readmission rate.

A high iPTH level implicates increased bone resorption. ${ }^{20}$ In patients with $\mathrm{CKD}$, the relationship between iPTH and low bone mass may become more complex, with interference by many factors. Since the bones of patients with CKD may be resistant, the iPTH level must be twofold to threefold higher than the normal range to maintain normal turnover of bone. ${ }^{13}$ However, there were no differences in levels between readmission and nonreadmission patients in this study. Therefore, iPTH could not be a contributing factor in readmissions.

Osteoporosis is frequently noted in patients with CKD, so we need to put in more effort to prevent bone loss. Since bone loss is progressive and silent, it is important to monitor bone mass in patients with CKD. Low bone mass increases the risk of fracture, so the clinician should keep alertness even if only osteopenia in CKD patients. Before antiosteoporotic therapy, we should routinely measure BMD to identify those at high risk for fractures.

Vertebral fractures have been associated with increased mortality. ${ }^{21}$ The mortality risk increases with vertebral fractures in elderly patients of all ages and ethnicities, and in both sexes. The difference in mortality rates between patients with a fracture and controls is higher than previously reported, even after controlling for comorbidities. ${ }^{22}$ In patients with $\mathrm{CKD}$, the risk of osteoporosis is higher, and the use of denosumab therapy will decrease fracture risk and mortality. In this study $12(41 \%)$, readmission was due to spine refracture.

Since the hip and FN are predominantly a measure of cortical bone while the LS predominantly trabecular bone, the main benefit of denosumab is the effect on trabecular bone, which increase bone density of LS and decrease risk of future fracture and readmission rate.

Table 2 Multivariable analysis of the ORs for readmission

\begin{tabular}{|c|c|c|c|c|c|}
\hline Variables & Regression coefficient & SE & Wald & $\mathrm{p}$ Value & OR $(95 \% \mathrm{Cl})$ \\
\hline Age (years) & -0.001 & 0.033 & 0.001 & 0.971 & 0.998 (0.936 to 1.065$)$ \\
\hline Gender & -0.317 & 0.739 & 0.183 & 0.668 & 0.728 (0.171 to 3.102$)$ \\
\hline Body mass index $\left(\mathrm{kg} / \mathrm{m}^{2}\right)$ & -0.037 & 0.073 & 0.259 & 0.611 & 0.963 (0.836 to 1.111$)$ \\
\hline Spine fracture (number) & 0.12 & 0.188 & 0.404 & 0.525 & 1.127 (0.779 to 1.631$)$ \\
\hline BMD (lumbar) & -0.673 & 0.248 & 7.369 & 0.007 & 1.960 (1.206 to 3.189$)$ \\
\hline BMD (total hip) & -0.336 & 0.548 & 0.377 & 0.539 & 0.714 (0.244 to 2.090$)$ \\
\hline BMD (femoral neck) & -0.165 & 0.587 & 0.079 & 0.779 & 0.847 (0.269 to 2.677$)$ \\
\hline Neurological disease & 0.763 & 0.69 & 1.222 & 0.269 & 2.143 (0.555 to 8.289$)$ \\
\hline Diabetes mellitus & 0.367 & 0.623 & 0.348 & 0.556 & 1.443 (0.426 to 4.897$)$ \\
\hline Hypertension & 0.151 & 0.639 & 0.056 & 0.813 & $1.163(0.333$ to 4.067$)$ \\
\hline Hyperlipidemia & 0.857 & 1.049 & 0.667 & 0.414 & 2.355 (0.301 to 18.405$)$ \\
\hline Cardiovascular disease & 0.117 & 0.691 & 0.029 & 0.865 & $1.124(0.290$ to 4.357$)$ \\
\hline Pulmonary disease & -0.116 & 0.861 & 0.018 & 0.893 & 0.890 (0.165 to 4.813$)$ \\
\hline Liver disease & -0.705 & 0.832 & 0.717 & 0.397 & 0.4941 (0.097 to 2.526$)$ \\
\hline
\end{tabular}


There are several limitations to this study. Readmission was identified on the basis of medical records during follow-up after varying time periods. We were not able to obtain all readmission data for all patients during follow-up and could not draw conclusions regarding a correlation between clinical symptoms and readmission. Second, this was a single-center study with a small patient population and a retrospective design. Therefore, we did not have complete data, such as the use of vitamin D and calcium supplements. These limitations restrict the generalizability of the results.

\section{CONCLUSION}

Osteoporosis in patients with CKD is associated with a high risk of readmission. The best predictor was $\mathrm{T}$ score at the LS. A lower T score (especially $<-3$ ) was associated with a higher probability of fracture readmission. Aggressive medical attention is needed to decrease a future trend toward fracture readmissions.

Acknowledgements The authors acknowledge Kaohsiung Chang Gang Memorial Hospital for afford the study data.

Contributors B-CC designed and performed the research; and Y-CC analyzed the data and wrote the final article.

Competing interests None declared.

Ethics approval This retrospective study was approved by the Institutional Review Board of our hospital. Informed consent was waived because of the retrospective nature of this study.

Provenance and peer review Not commissioned; externally peer reviewed.

Data sharing statement The data set supporting the conclusion of this article is available on request from the corresponding author.

Open Access This is an Open Access article distributed in accordance with the Creative Commons Attribution Non Commercial (CC BY-NC 4.0) license, which permits others to distribute, remix, adapt, build upon this work noncommercially, and license their derivative works on different terms, provided the original work is properly cited and the use is non-commercial. See: http:// creativecommons.org/licenses/by-nc/4.0/

\section{REFERENCES}

1 Alem AM, Sherrard DJ, Gillen DL, et al. Increased risk of hip fracture among patients with end-stage renal disease. Kidney Int 2000;58:396-9.

2 Gal-Moscovici A, Sprague SM. Osteoporosis and chronic kidney disease. Semin Dial 2007;20:423-30.

3 Stehman-Breen CO, Sherrard DJ, Alem AM, et al. Risk factors for hip fracture among patients with end-stage renal disease. Kidney Int 2000;58:2200-5.
4 Baszko-Blaszyk D, Grzegorzewska AE, Horst-Sikorska W, et al. Bone mass in chronic renal insufficiency patients treated with continuous ambulatory peritoneal dialysis. Adv Perit Dial 2001;17:109-13.

5 Yamaguchi T, Kanno E, Tsubota J, et al. Retrospective study on the usefulness of radius and lumbar bone density in the separation of hemodialysis patients with fractures from those without fractures. Bone 1996; 19:549-55.

6 Moe S, Drueke T, Cunningham J, et al. Definition, evaluation, and classification of renal osteodystrophy: a position statement from Kidney Disease: Improving Global Outcomes (KDIGO). Kidney Int 2006;69:1945-53.

7 Lacey DL, Boyle WJ, Simonet WS, et al. Bench to bedside: elucidation of the OPG-RANK-RANKL pathway and the development of denosumab. Nat Rev Drug Discov 2012;11:401-19.

8 Boyle WJ, Simonet WS, Lacey DL. Osteoclast differentiation and activation. Nature 2003:423:337-42.

9 Kostenuik PJ, Nguyen HQ, McCabe J, et al. Denosumab, a fully human monoclonal antibody to RANKL, inhibits bone resorption and increases BMD in knock-in mice that express chimeric (murine/human) RANKL. J Bone Miner Res 2009:24:182-95.

10 Delmas PD. Clinical potential of RANKL inhibition for the management of postmenopausal osteoporosis and other metabolic bone diseases. I Clin Densitom 2008;11:325-38.

11 Bone HG, Bolognese MA, Yuen CK, et al. Effects of denosumab on bone mineral density and bone turnover in postmenopausal women. J Clin Endocrinol Metab 2008;93:2149-57.

12 McClung MR, Lewiecki EM, Cohen SB, et al. Denosumab in postmenopausal women with low bone mineral density. N Engl J Med 2006;354:821-31.

13 McClung MR, Lewiecki EM, Geller ML, et al. Effect of denosumab on bone mineral density and biochemical markers of bone turnover: 8-year results of a phase 2 clinical trial. Osteoporos Int 2013;24:227-35.

14 Cunningham J, Sprague SM, Cannata-Andia J, et al. Osteoporosis in chronic kidney disease. Am J Kidney Dis 2004;43:566-71.

15 Hsu CC, Hwang SJ, Wen CP, et al. High prevalence and low awareness of CKD in Taiwan: a study on the relationship between serum creatinine and awareness from a nationally representative survey. Am J Kidney Dis 2006;48:727-38

16 Chen YR, Yang Y, Wang SC, et al. Multidisciplinary care improves clinical outcome and reduces medical costs for pre-end-stage renal disease in Taiwan. Nephrology (Carlton) 2014;19:699-707.

17 van Geel TA, van Helden S, Geusens PP, et al. Clinical subsequent fractures cluster in time after first fractures. Ann Rheum Dis 2009;68:99-102.

18 Cummings SR, San Martin J, McClung MR, et al. Denosumab for prevention of fractures in postmenopausal women with osteoporosis. N Engl J Med 2009:361:756-65.

19 Gabay C, Ruedin P, Slosman D, et al. Am J Nephrol 1993;13(2):115-23.

20 Kanis JA, Seeman $E$, Johnell 0 , et al. The perspective of the international osteoporosis foundation on the official positions of the international society for clinical densitometry. J Clin Densitom 2005;8:145-7.

21 Puisto V, Rissanen $\mathrm{H}$, Heliovaara $\mathrm{M}$, et al. Mortality in the presence of a vertebral fracture, scoliosis, or Scheuermann's disease in the thoracic spine. Ann Epidemiol 2008;18:595-601.

22 Lau E, Ong K, Kurtz S, et al. Mortality following the diagnosis of a vertebral compression fracture in the Medicare population. J Bone Joint Surg Am 2008;90:1479-86. 